
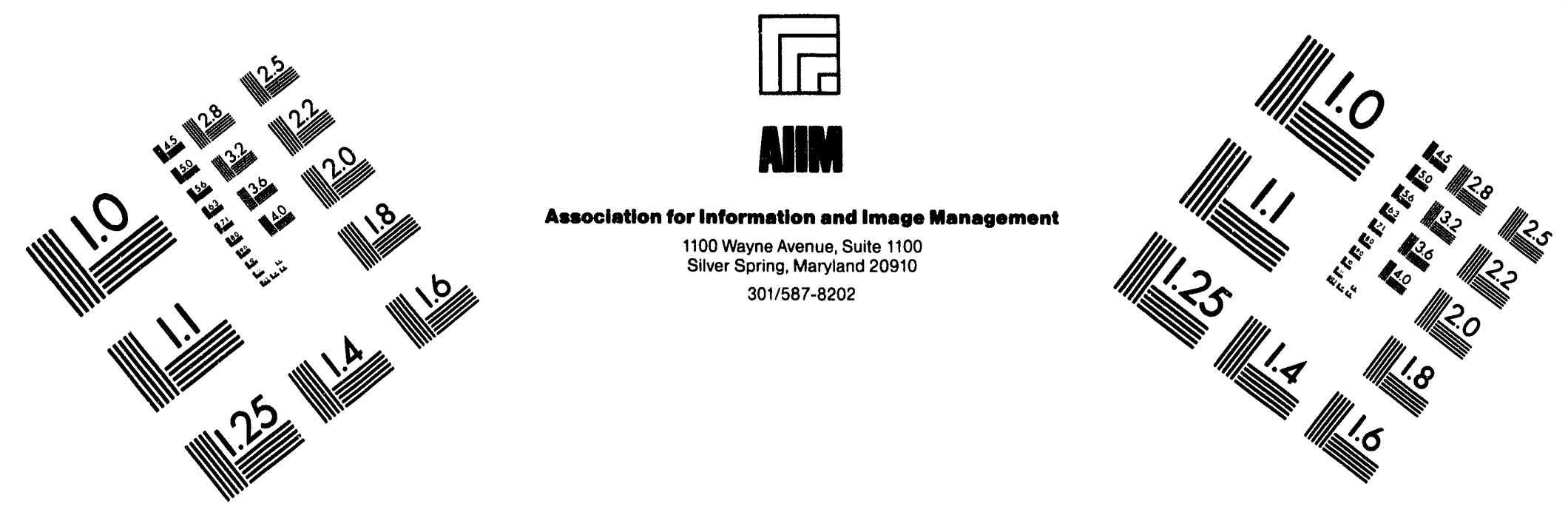

\title{
Centimeter
}

$\begin{array}{llllllllllllllll}1 & 2 & 3 & 4 & 5 & 6 & 7 & 8 & 9 & 10 & 11 & 12 & 13 & 14 & 15 & \mathrm{~mm}\end{array}$ Inches

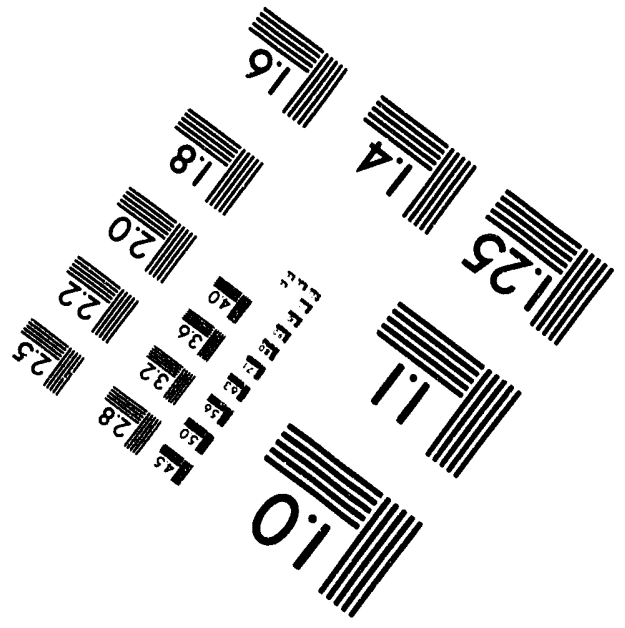

MANUFACTURED TO AIIM STANDARDS BY APPLIED IMAGE, INC.

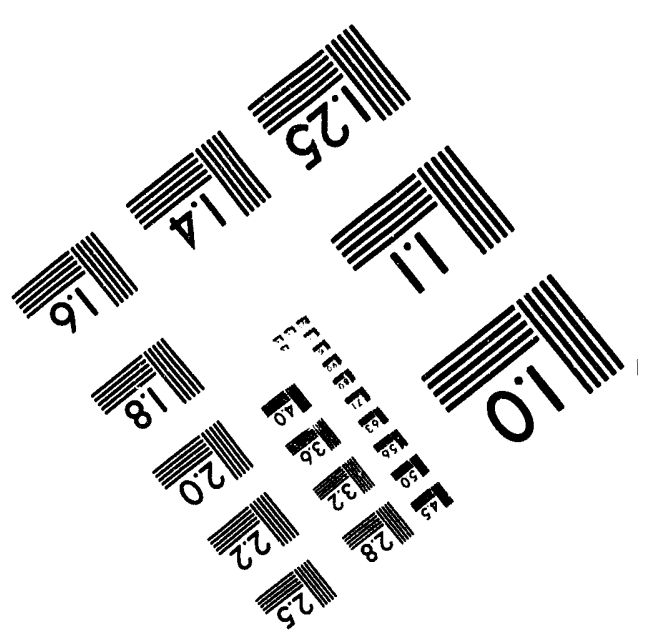



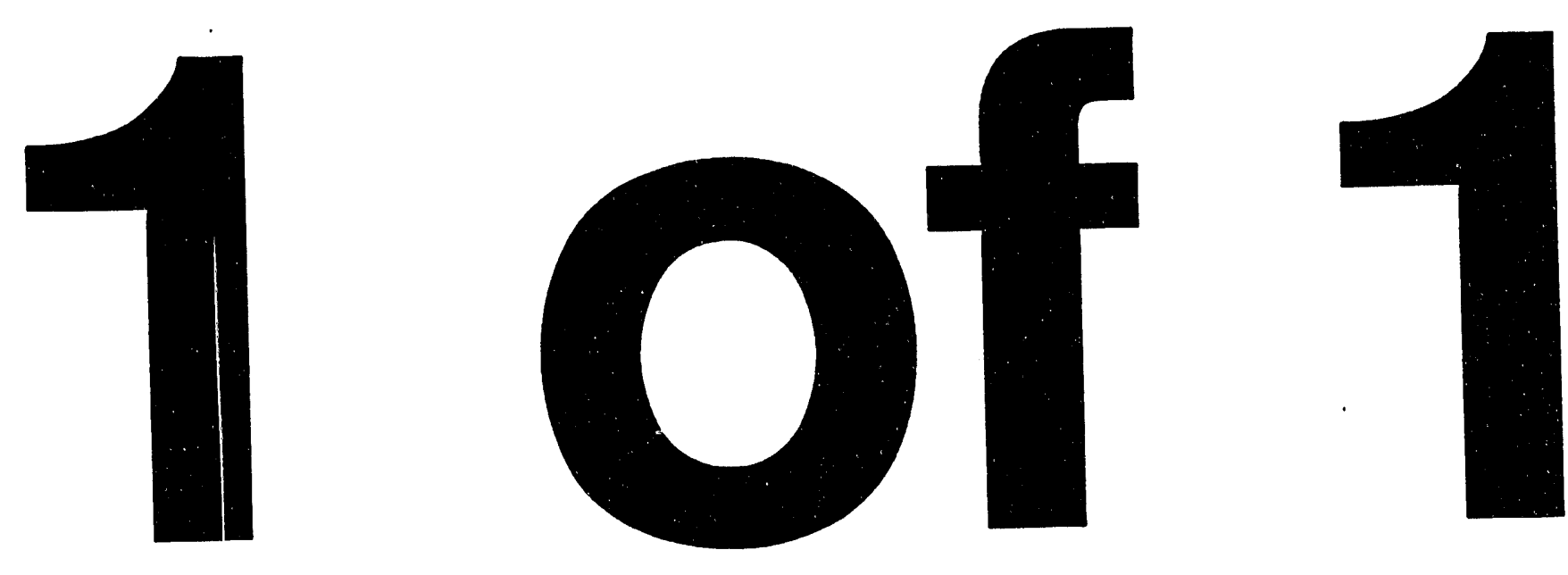


\section{Geometrical Barriers in Type II Superconductors}

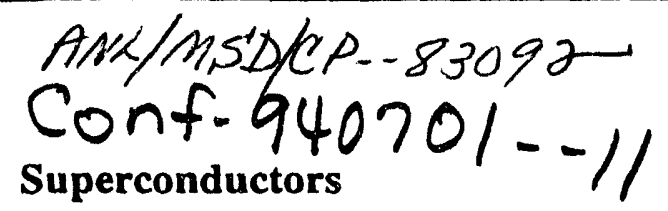

E. Zeldov, ${ }^{1}$ A.I. Larkin, ${ }^{1,2}$ M. Konczykowski, ${ }^{3}$ B. Khaykovich, ${ }^{1}$ D. Majer, ${ }^{2}$

V.B. Geshkenbein, ${ }^{1,2}$ and V.M. Vinokur ${ }^{4}$

1Weizmann Institute of Science, 76100 Rehovot, Israei

${ }^{2}$ L.D. Landau Institute for Theoretical Physics, 117940 Moscow, Russia

${ }^{3}$ Laboratoire des Solides Irradies, Ecole Polytechnique, 91128 Palaiseau, France

${ }^{4}$ Materials Science Division, Argonne National Laboratory, ${ }^{*}$ Argonne, IL 60439

Proceedings of the 4th International Conference on Materials and Mechanisms of Superconductivity, High-Temperature Superconductors (M ${ }^{2} S-H T S C$ IV), Grenoble, France, July 5-9, 1994, edited by P. Wyder, to be published in PHYSICA C

\section{DISCLAIMER}

This report was prepared as an account of work sponsored by an agency of the United States Government. Neither the United States Government nor any agency thereof, nor any of their employees, makes any warranty, express or implied, or assumes any legal liability or responsibility for the accuracy, completeness, or usefulness of any information, apparatus, product, or process disclosed, or represents that its use would not infringe privately owned rights. Reference herein to any specific commercial product, process, or service by trade name, trademark, manufacturer, or otherwise does not necessarily constitute or imply its endorsement, recommendation, or favoring by the United States Government or any agency thereof. The views and opinions of authors expressed herein do not necessarily state or reflect those of the United Staies Government or any agency thereof.

\footnotetext{
*Work supported by the U.S. Department of Energy, BES-Materials Sciences under contract \#W-31-109-ENG-38.
} 


\title{
Geometrical Barriers in Type II Superconductors
}

\author{
E. Zeldov(1), A. I. Larkin(2),(1), M. Konczykowski( ${ }^{(3)}$, B. Khaykovich ${ }^{(1)}$, D. Majer(1),
} V. B. Geshkenbein ${ }^{(2),(1)}$, and V. M. Vinokur ${ }^{(4)}$

(1)Weizmann Institute of Science, 76100 Rehovot, Israel, (2) L. D. Landau Institute for Theoretical Physics, 117940 Moscow, Russia, ${ }^{(3)}$ Laboratoire des Solides Irradies, Ecole Polytechnique, 91128 Palaiseau, France, ${ }^{(4)}$ Argonne National Laboratory, Argonne, Illinois 60439, USA

Vortex penetration in thin flat samples in perpendicular fields is governed by geometrical barrier effect. The main features of this novel barrier is vortex accumulation in the center of the sample and irreversible magnetization in the absence of bulk pinning. The developed theoretical model is confirmed by experimental results.

The magnetization behavior of thin superconducting samples is usually modeled by approximation to an elliptical cross-section [1]. This common procedure fails to resolve some important aspects of vortex dynamics which are specific to thin flat samples in perpendicular magnetic fields. In particular, in absence of pinning and surface barriers, a reversible magnetization is expected in elliptical samples. In contrast, flat samples exhibit unique hysteretic behavior which is the result of geometrical barriers as discussed below.

Consider a long thin superconducting strip of rectangular cross-section of width $2 W(-W<$ $x<W)$ and thickness $d(-d / 2<z<d / 2$, and $d<<W$ ) exposed to applied field $H_{a} \| z$. In absence of bulk pinning the resulting shielding current density averaged over the sample thickness is given by [2]

$$
\begin{aligned}
& J_{y}(x)= \\
& \begin{cases}0, & 0<x<b, \\
-\frac{2 J_{E}^{0}}{x} \arctan \sqrt{\frac{\left(W^{2}-e^{2}\right)\left(x^{2}-b^{2}\right)}{\left(W^{2}-b^{2}\right)\left(e^{2}-x^{2}\right)}}, & b<x<e, \\
-J_{E}^{0}, & e<x<W,\end{cases}
\end{aligned}
$$

and $-J_{y}(x)$ for $x<0$. The edge current $J_{E}^{0}$ is determined by the following consideration. In increasing applied field the vortices initially cut through the sharp rims of the sample without complete penetration and thus effectively round off the curvature of the edges on the order of $d / 2$. As a result, the vortex energy increases gradually from zero to a maximum value of $c_{0} d$ at $W-|x| \simeq d / 2$. At steady state conditions the resulting force due to this potential gradient has to be balanced by Lorentz force of the shielding currents, $J_{E}^{0} \phi_{0} d / c$, and hence $J_{E}^{0} \simeq 2 c \epsilon_{0} / \phi_{0} d=$ $c H_{c 1} / 2 \pi d$. $\phi_{0}$ is the flux quantum and $\epsilon_{0}$ the vortex line energy. The corresponding transverse field profile inside and outside the sample is

$$
\begin{aligned}
& B_{z}(x)= \\
& \left\{\begin{array}{rr}
\frac{2 H_{a 1}}{\pi} \ln \frac{\sqrt{\left|e^{2}-x^{2}\right|\left(W^{2}-b^{2}\right)}+\sqrt{1 b^{2}-x^{2} \mid\left(W^{2}-e^{2}\right)}}{\sqrt{\left(e^{2}-b^{2}\right)\left|W^{2}-x^{2}\right|}} \\
|x|<b \text { or }|x|>e, \\
0, \quad b<|x|<e,
\end{array}\right.
\end{aligned}
$$

with the resulting magnetization per unit volume $M=-\frac{H_{c 1}}{4 \pi W d} \sqrt{\left(W^{2}-b^{2}\right)\left(W^{2}-e^{2}\right)}$.

The parameters $b$ and $e$ are determined by the applied field

$H_{a}=\frac{2 H_{c 1}}{\pi} \ln \frac{\sqrt{W^{2}-b^{2}}+\sqrt{W^{2}-e^{2}}}{\sqrt{e^{2}-b^{2}}}$

and by the history of the magnetic cycle as follows. As the field is initially increased, $b=0$ and the vortices penetrate partially only into the edge regions $e<|x|<W$. The resulting current and field profiles are similar to the case in which vortex penetration is limited by bulk pinning [3] with $J_{E}^{0}$ replacing $J_{c}$. This situation holds as long as $e>W-d / 2$ and hence $H_{a}<H_{p} \simeq\left(2 H_{\mathrm{cl}} / \pi\right) \sqrt{d / W}$. Above the penetration field $e=W-d / 2$ and the penetrating vortices are dragged by the extended shielding currents towards the center, where a vortex-filled region of width $2 b$ is formed as shown in Fig. 1. The 


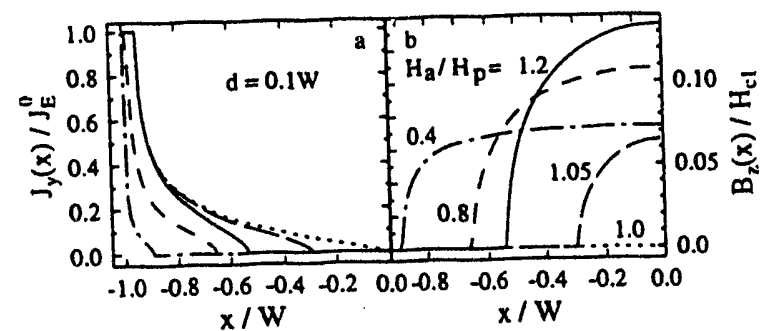

Figure 1. Calculated current (a) and field (b) profiles in half of a strip in ascending $\left(H_{a} / H_{p}=1\right.$, $1.05,1.2)$ and descending $(0.8,0.4)$ fields. Maximum applied field is $H_{\max }=1.2 H_{p}$.

resulting apparent flux growth from the center of the sample is a unique consequence of the flat geometry. This situation, however, is not reversible since on decreasing field the central vortices are effectively trapped by the shielding currents. In this case both $b$ and $e$ increase with the declining field and their values are determined by $H_{a}$ and the condition of constant trapped flux inside the sample. Fig. 1 shows the resulting current and field profiles, and the irreversible magnetization loops are shown in Fig. 2. Note that in contrast to bulk $J_{c}$ the edge current $J_{E}^{0}$ does not change polarity as field decreases and hence $M<0$ also on the descending branch. The trapped vortices exit the sample when $b \simeq W-d / 2$. At high fields a reversible magnetization is obtained when $e=W-d / 2 \simeq b$ at $H_{a}>H_{I L} \sim 1.5 H_{c 1}$.

We have measured the $B_{z}(x)$ profiles in $\mathrm{Bi}_{2} \mathrm{Sr}_{2} \mathrm{CaCu}_{2} \mathrm{O}_{8}$ crystals using sensitive microscopic GaAs/AlGaAs Hall-sensor arrays. Fig. 3 shows the experimental field profiles at $T=50 \mathrm{~K}$ in ascending applied field. For $H_{a}<450$ no vortices penetrate into the sample. As field increases, the flux build-up in the center of the sample and the expansion of vortex-filled region are clearly visible. The observed increase of the background field inside the crystal close to the edge is the result of small finite gap between crystal surface and the sensors.

We are grateful H. Shtrikman for GaAs heterostructures and to N. Motohira for BSCCO crystals. This work was supported by the Israeli Ministry of Science and the Arts, by the Philip M. Klutznick Fund, by MINERVA foundation, and by France-Israel cooperation program PICS 112. VMV acknowledges the support through US

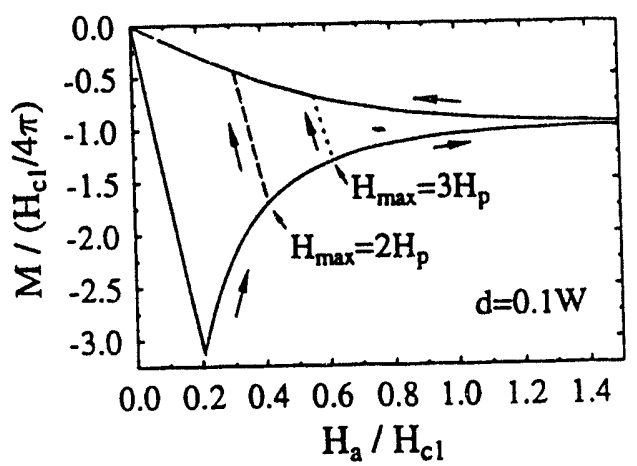

Figure 2. Calculated magnetization loops of a strip showing irreversible behavior due to geometrical barrier.

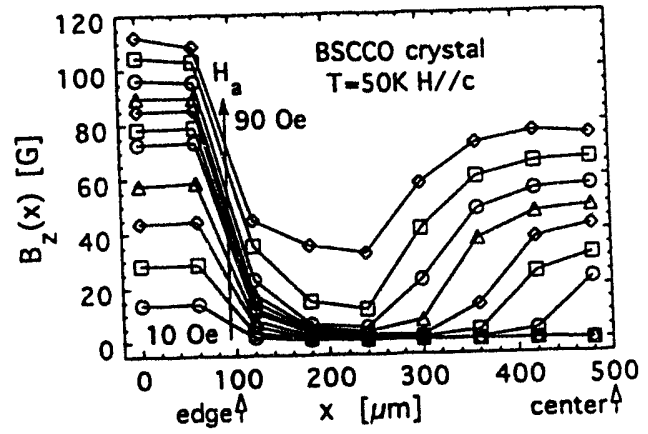

Figure 3. Experimental local field profiles in ascending $H_{a}$. Hall-sensor array consisted of nine $30 \times 30 \mu \mathrm{m}^{2}$ elements $30 \mu \mathrm{m}$ apart. The connecting lines are guide to the eye.

Department of Energy, BES-Materials Sciences, under contract No. W-31-109-ENG-38.

\section{REFERENCES}

1. R.P. Huebener, R.T. Kampwirth, and J.R. Clem, J. Low Temp. Phys 6, 275 (1972).

2. E. Zeldov, A.I. Larkin, V.B. Geshkenbein, M. Konczykowski, D. Majer, B. Khaykovich, V.M. Vinokur, and H. Shtrikman (unpublished).

3. E. Zeldov, J.R. Clem, M. McElfresh, and M. Darwin, Phys. Rev. B 49, 9802 (1994); E.H. Brandt and M.V. Indenbom, Phys. Rev. B 48, 12893 (1993); A.I. Larkin and Yu.N. Ovchinnikov, Zh. Eksp. Teor. Fiz. 61, 1221 (1971) [Sov. Phys. JETP 34, 651 (1972)]. 

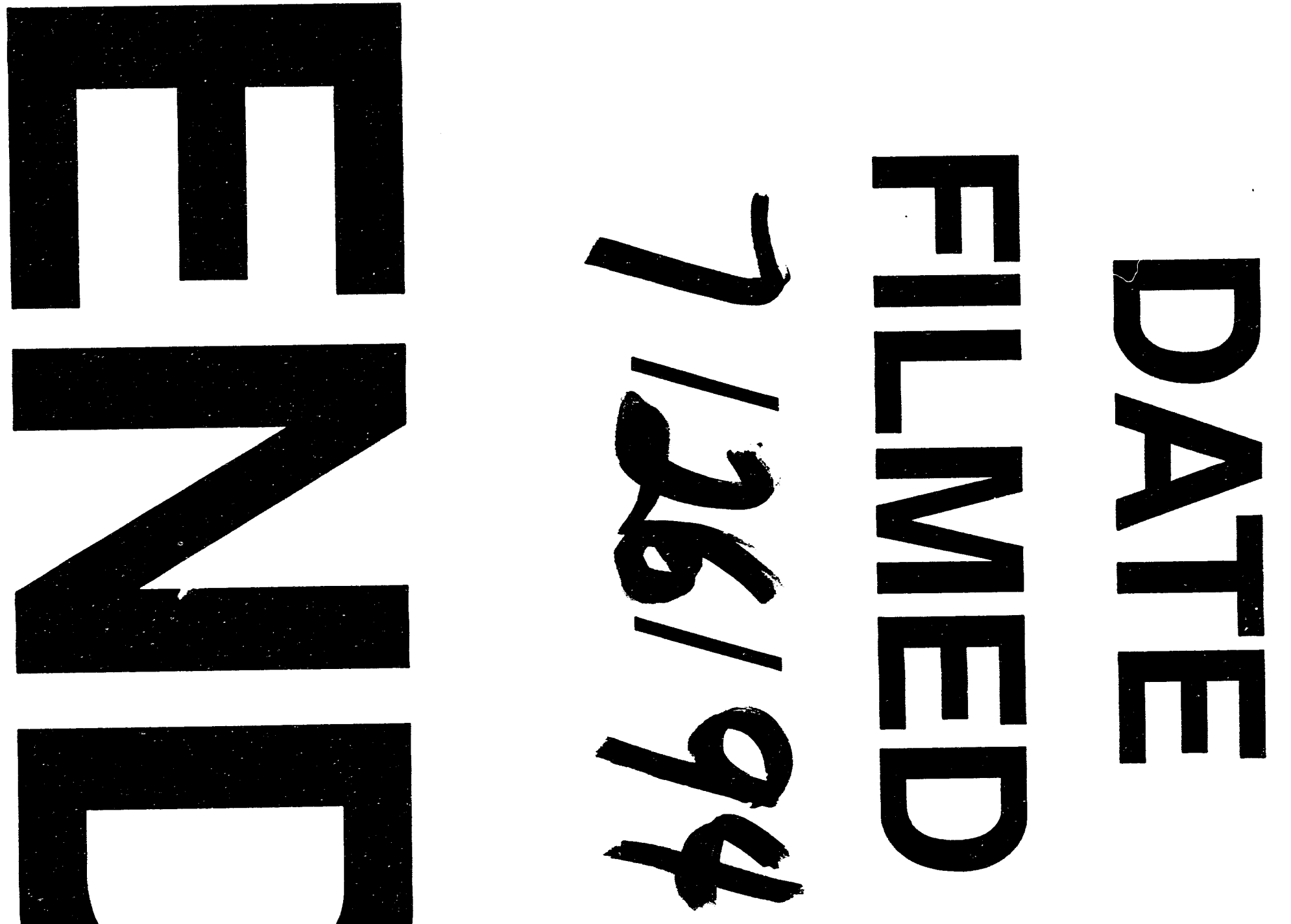
